\title{
HEMISECTION FOR THE TREATMENT OF ADVANCED ENDODONTIC PERIODONTAL LESION
}

\author{
Dr. Ashu Gupta ${ }^{1}$ Dr. Saroj Thakur ${ }^{2}$ Dr. Rambhika Thakur ${ }^{3}$ \\ ${ }^{1 .}$ Professor \& H.O.D., Department of Conservative Dentistry, H.P.G.D.C \& H, Shimla, Himachal Pradesh (India) \\ 2. Lecturer Department of Conservative Dentistry, H.P.G.D.C \& H, Shimla, Himachal Pradesh (India) \\ ${ }^{3 .}$ Lecturer Department of Periodontology, M.N.D.A.V. Dental College Solan, Himachal Pradesh (India) \\ Corresponding Author: \\ ${ }^{1}$ Mobile: 919418470020 Email: guptaashumoni@gmail.com
}

Received :

$16^{\text {th }}$ Oct, 2013

Accepted:

$20^{\text {th }}$ Dec, 2013

Available online:

$28^{\text {th }}$ Dec, 2013

\section{ABSTRACT}

The removal of a root and the overlaying anatomic crown is referred to as a hemisection. A case with a complain of pain and bone loss till the apex of distal root of 36 presented in the department of Endodontics, Government dental college, Shimla. The patient had increased desire to maintain the natural dentition. The decision was taken to hemi-sect the distal root, as mesial bone and furcation bone was relatively unaffected. After phase I therapy, periodontal flap was reflected, area debrided and distal root was resected. This article describes a procedure of hemisection in mandibular molar and its subsequent restoration. The key to long term success appear to be thorough diagnosis, selection of patients with good oral hygiene and careful surgical and restorative management.

Key words: Periodontitis, Permanent dental restoration, Surgery.

\section{INTRODUCTION}

In 1867, Magitot reported on the complete removal (root resection or amputation) of molar roots. ${ }^{1}$ However, Farrar, in 1884 is often cited as the first author and clinician to detail the resective techniques for the radical removal by amputation of any portions of the roots of the teeth that can be of no further use. ${ }^{2}$ Through root resection therapy furcation involved molars can be converted to non-furcated single root teeth and provide a favorable environment for oral hygiene for patients and clinicians. In a recent article, Minsk and Polson suggested that root resection can be a valuable procedure when the tooth in question has a very high strategic value or when there are specific problems that cannot be solved by other therapeutic procedures. Teeth in proximity to anatomic landmarks, such as the maxillary sinus, inferior alveolar canal, can be treated safely by root resection therapy. In addition resected molars can be used for alveolar bone augmentation by orthodontic movement. This procedure represents a form of conservative dentistry, aiming to retain as much of the original tooth structure as possible. ${ }^{3}$ Hemisection (removal of one root) involves removing significantly compromised root structure and the associated coronal structure through deliberate excision. ${ }^{4}$
Appropriate endodontic therapy must be performed before these tooth modifications to avoid intrapulpal dystrophic calcification and postoperative tooth sensitivity. ${ }^{5}$ The furcation region is carefully smoothed, to allow proper cleansing and thus to prevent accumulation of plaque. ${ }^{5}$ Root fracture is the main cause of failure after hemisection, so occlusal modifications are required to balance the occlusal forces on the remaining root. ${ }^{6}$

\section{CASE REPORT}

A 34-year-old man reported to the dental clinic with the chief complaint of intermittent pain on the lower left side of the mouth since 4 months. Clinically over all gingival health appeared normal, Oral hygiene index- good (0.6), Plaque index- good (0.37). Over all periodontal status was good except the probing pocket depth was $10 \mathrm{~mm}$ around the distal root of 36 , grade II furcation involvement in 36, it was also sensitive to percussion. On radiographic examination, severe vertical bone loss was evident surrounding the distal root and involving the furcation area (Figure 1). The bony support of mesial root was completely intact. This case was diagnosed as Localized periodontitis with respect to 36 and 37 . The treatment 
consisted of phase I therapy and thorough scaling and root planning was done. Gingival and periodontal status was reevaluated after 2 weeks.

Phase II (Surgical phase): A decision was taken that the distal root should be hemisected after completion of endodontic therapy of the tooth. The tooth could be saved as mesial root had good bone and furcation area had reasonably good bone support. The working length was determined and the canals were biomechanically prepared using crown-down technique using protaper rotary instuments as per manufacturer instructions. Mesiobuccal and mesiolingual canals were prepared upto F3 ( ISO 30 no.). The canals were obturated with lateral condensation method and the chamber was filled with amalgam to maintain a good seal and allow interproximal area to be properly contoured during surgical separation (Figures 2, 3). Under local anesthesia, mucoperiosteal flap was reflected after giving a crevicular incision from first premolar to second molar (Figure 4. Upon reflection of the flap, the bony defect along the distal root became quite evident. All chronic inflammatory tissue was removed with currettes to expose the bone. The vertical cut method was used to resect the crown. A long shank tapered fissure carbide bur was used to make vertical cut toward the bifurcation area (Figure 5). The distal root was extracted and the socket was irrigated adequately with sterile saline to remove bony chips and amalgam debris (Figures 6, 7). The flap was then repositioned and sutured with $3 / 0$ black silk sutures.

Phase III (Restorative phase): The occlusal table was minimized to redirect the forces along the long axis of the mesial root. Hemisected molar was restored with full coverage cast restoration (Figures 8, 9).

Phase IV (Maintenance phase): Patient had been followed up since with regular recall visits and oral prophylaxis. He had good masticatory efficiency with the restoration was very happy with the treatment outcome.

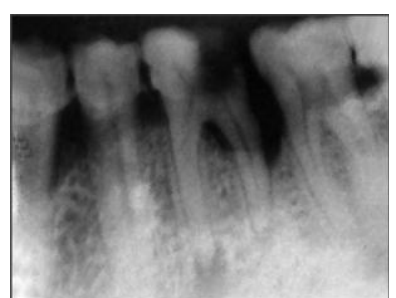

Figure 1: Preoperative IOPA of tooth No. 36
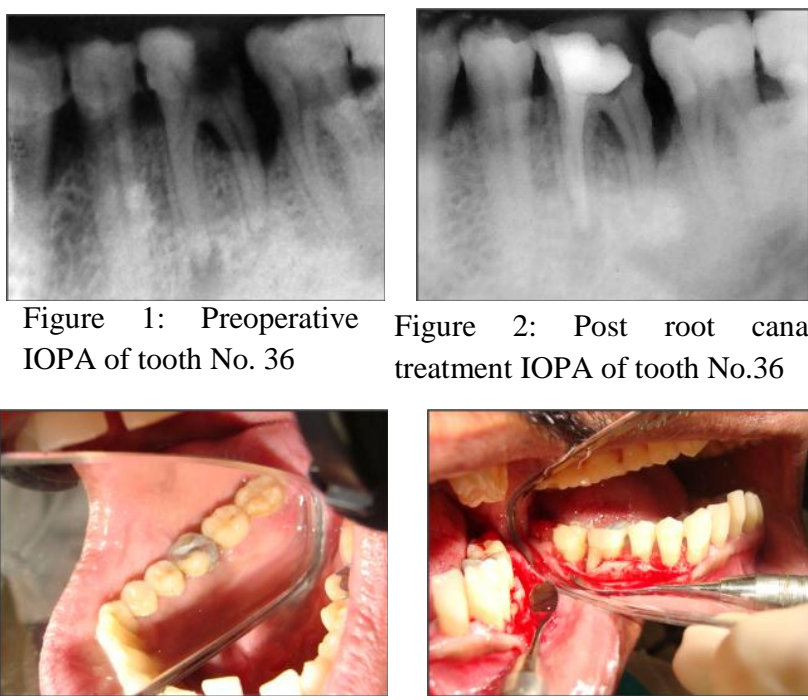

Figure 3: Intra-oral lingual view of tooth No.36

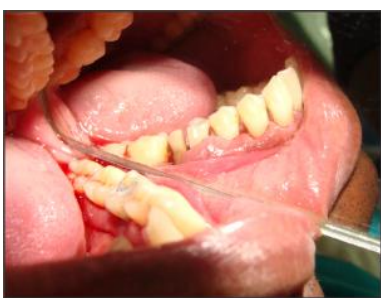

Figure 5: Hemisection of tooth No.36

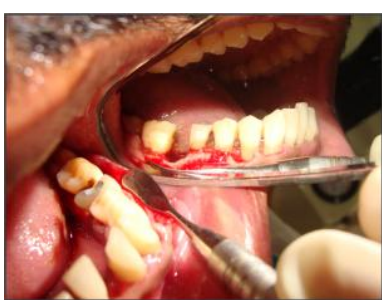

Figure 7: Mesial half segment of tooth No.36

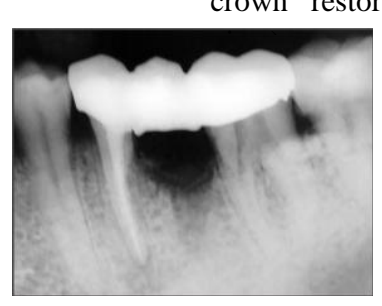

Figure 9: Postoperative IOPA

\section{DISCUSSION}

Implant therapy is a predictable option with good functionality. ${ }^{7}$ However, in this case, the patient chose an alternative treatment because of financial considerations and his desire to retain the natural tooth. The smaller size of the occlusal tables, undercontouring of the embrasure spaces and ensuring that 
the crown margin encompasses the furcation are all factors in the high success rates observed with hemisection therapy. ${ }^{5}$ According to Buhler et al, hemisection should be considered before every molar extraction, because this procedure can provide a good absolute biological cost savings with good long-term success. In addition, he reported that the failure rates of single-tooth alloplastic (titanium) implants and hemisections are not substantially different. ${ }^{4}$ The decision of hemisecting the tooth should be based on the extent and pattern of bone loss, root trunk and root length, ability to eliminate the osseous defects and endodontic - restorative consideration ${ }^{8}$, consideration should be given to the morphology, clinical length and shape of the roots of a multirooted tooth. ${ }^{9}$ It is important to take into account the divergence of the roots while making a case selection. Affected teeth with roots spread apart facilitate the clinician's ability to carry out root resection. Teeth with closely approximated or fused roots are not good choices to receive hemisection therapy. ${ }^{9}$ Hemisection depends on angulation and position of the tooth in the arch. A molar that is buccally, lingually, mesially or distally titled, cannot be resected. Hemisection has been used successfully to retain teeth with furcation involvement. In the present case the above mentioned indication for case selection in performing hemisection was optimum as the roots were not closely approximated or fused. The tooth had to be endodontically treated before hemisection. In situations when resection periodontal therapy is decided, initiation of conventional endodontic treatment before therapy simplifies the surgical procedure. This is because tooth preparation can invade the pulp chamber and jeopardize control of the coronal seal of the endodontic access opening complicating the completion of endodontic therapy. ${ }^{2}$ However, there are few disadvantages associated with it. Root surfaces that are reshaped by grinding in the furcation or at the site of hemisection are more susceptible to caries. In addition, when the tooth has lost part of its root support, it will require a restoration to permit it to function independently or to serve as an abutment for a splint or bridge. ${ }^{2}$

The prognosis for hemisection is the same as for routine endodontic procedures provided that case selection has been correct, the endodontics has been performed adequately, and the restoration is of an acceptable design relative to the occlusal and periodontal needs of the patient. The prognosis of root resected molars may not be as poor as previously believed. ${ }^{10}$ Multirooted, periodontally involved molars can be maintained for long periods of time with hemisection. The large variation in success and failure reported by different authors is a reflection that roots resection and hemisection is a technique sensitive procedure. $^{10}$ One must be careful throughout the processes of case selection, and endodontic, periodontal, restorative and maintenance therapies. Critical analysis before reconstruction and regular reevaluation during maintenance period are crucial. $^{10}$ Langer et al reviewed records of 100 patients who had undergone root resection over a 10 years period. They reported a failure rate of $38 \%$, of which $15.8 \%$ occurred within the first 5 years after surgery. Most failures involved mandibular teeth and occurred for reasons other than inflammatory periodontal disease. ${ }^{11}$

There are only few long term studies on the survival of teeth after hemisection and root amputation published. These investigations often lack information about case selection and subsequent restoration. Furthermore there are conflicting data about the survival of the remaining fragment (3\% $38 \%$ for ten years.) and a wide range of reasons for failure of hemisection. ${ }^{12}$

Carnevale in his study on long term effects of root resective therapy suggested that it can be considered an effective measure to resolve periodontal problems of furcation defects. ${ }^{12}$ The data indicate that recurrent periodontal disease is not a major cause of the failure of these teeth. It was shown that such teeth can function successfully for long periods .Therefore, early and correct diagnosis is imperative, as delay will result in rapid loss of supporting bone and eventually tooth loss. ${ }^{12}$

\section{CONCLUSION}

Hemisection as a treatment option to conserve the tooth structure and use it as an abutment is still very relevant. The prognosis of root resected molars may not be as grim as previously believed. The decision of hemisecting the tooth should be based on the extent and pattern of bone loss, root trunk and root length, ability to eliminate the osseous defects and endodontic- restorative consideration. The data indicate that recurrent periodontal disease is not a major cause of the failure of these teeth. It was shown 
that such teeth can function successfully for long periods.

\section{REFERENCES}

1. Prinz H. Dental chronology. Philadelphia Lea \& Febiger, 1945;119

2. Farrar JN. Radical and heroic treatment of alveolar abscess by amputation of roots of teeth. Dent Cosmos 1884;26:7981 .

3. Kost WJ, Stakiw JE. Root amputation and hemisection. J Can Dent Assoc 1991; 57(1):42-5.

4. Bühler H. Survival rates of hemisected teeth: an attempt to compare them with survival rates of alloplastic implants. Int J Periodontics Restorative Dent1994; 14(6):536-543.

5. Rapoport RH, Deep P. Traumatic hemisection and restoration of a maxillary first premolar: a case report. Gen Dent 2003; 51(4):340-342.

6. Kryshtalskyj E. Root amputation and hemisection. Indication, technique and restoration. J Can Dent Assoc 1986; 52(4):307-8.
7. Bashutski JD, Wang HL. Common implant esthetic complications. Implant Dent 2007; 16(4):340-8

8. Newman, Takei, Fermin A Carranza. Furcation: Involvement and treatment; Willium $\mathrm{F}$ IN: Clinical Periodontology, X edition, WB Saunder's Co, 200:996.

9. Kurtzman GM, Silverstein LH, Shatz PC. Hemisection as an alternative treatment for vertically fractured mandibular molars. Compend Contin Educ Dent 2006; 27(2):126-9.

10. Baston $\mathrm{CH}$, Ammons WF, Persson R: Long term evaluation of root resected molars: A retrospective study:Int J Periodont Restor Dent 1996;16(3):207.

11. Carnevale G, Difebo G, Toyelli MP: A retrospective analysis of the periodontal- Prosthetic treatment of molars with interradicular lesions; Int J Periodont Restor Dent1991; 11:189-205.

12. Carnevale G, Pontoricro R, Di febo G. Long term effect of root resective therapy in furcation involved molars.-A 10 year longitudinal study J Clin. Periodontol 1998;25:209- 14. 